\title{
ON THE GRADIENT OF SOLID HARMONIC POLYNOMIALS*
}

\author{
BY \\ G. SZEGÖ
}

To Leopold Fejér

on his 60th birthday, February 9, 1940.

\section{INTRODUCTION}

In 1912 S. Bernstein proved a theorem on trigonometric polynomials which has been considered by various authors during subsequent years. $\dagger$ For our later purposes it is convenient to formulate this theorem in terms of harmonic polynomials as follows:

I. Let $u(x, y)$ be a real harmonic polynomial of the nth degree which satisfies the inequality $|u(x, y)| \leqq 1$ in the unit circle $x^{2}+y^{2} \leqq 1$. If $l$ denotes the positive tangential direction at an arbitrary point $(x, y)$ of the unit circle $x^{2}+y^{2}=1$, we have

$$
\left|\frac{\partial u}{\partial l}\right| \leqq n, \quad x^{2}+y^{2}=1 .
$$

The equality sign holds only if $u(x, y)=\cos n\left(\phi-\phi_{0}\right)$ for $x^{2}+y^{2}=1$, where $(1, \phi)$ are the polar coordinates of the point with the cartesian coordinates $(x, y)$ on the unit circle, and $\phi_{0}$ is arbitrary real.

Ten years ago I proved the following theorem, which is more informative than I:

II. Let $u(x, y)$ be a real harmonic polynomial of the nth degree which satisfies the inequality $|u(x, y)| \leqq 1$ in the unit circle $x^{2}+y^{2} \leqq 1$. Then

$$
|\operatorname{grad} u|=\left(u_{x}^{2}+u_{y}^{2}\right)^{1 / 2} \leqq n, \quad x^{2}+y^{2} \leqq 1 .
$$

The equality sign holds for the same polynomials as in I; it holds only for $x^{2}+y^{2}$ $=1$ if $n>1$.

In the present investigation I am dealing with the three-dimensional analogue of the last theorem. The following result holds:

* Presented to the Society, September 8, 1939; received by the editors September 6, 1939.

† See the literature quoted in the introduction of the paper referred to in the next footnote.

† G. Szegö, Über einen Satz des Herrn Bernstein, Schriften der Königsberger Gelehrten Gesellschaft, 1928, pp. 59-70. See also S. Bernstein, Sur un theorème de M. Szegö, Prace MatematycznoFizyczne, vol. 44 (1937), pp. 9-14. 
III. Let $u(x, y, z)$ be a real harmonic polynomial of the nth degree which satisfies the inequality $|u(x, y, z)| \leqq 1$ in the unit sphere $x^{2}+y^{2}+z^{2} \leqq 1$. We assume $n \geqq 4$. Then

$$
\begin{aligned}
&|\operatorname{grad} u|=\left(u_{x}^{2}+u_{y}^{2}+u_{z}^{2}\right)^{1 / 2} \\
& \leqq 2 n\left(\frac{1}{1}-\frac{1}{3}+\cdots+\frac{(-1)^{n-1}}{2 n-1}\right)+\left\{\begin{array}{r}
0 \text { if } n \text { even, } \\
-1 \text { if } n \text { odd }, \\
x^{2}+y^{2}+z^{2} \leqq 1 .
\end{array}\right.
\end{aligned}
$$

The equality sign holds only for the polynomial $u(x, y, z)$ whose boundary values on the unit sphere $x^{2}+y^{2}+z^{2}=1$ are of the form $\pm \cos n \gamma$; here $\gamma$ is the spherical distance of the variable point $(x, y, z)$ on the unit sphere from a fixed point $P_{0}$ of this sphere.

For these polynomials the equality sign holds only at $P_{0}$ and at the point diametral to $P_{0}$.

The bound $\rho_{n}$ in the inequality (3) is the precise one. We have $\rho_{n} \cong n \pi / 2$ as $n \rightarrow \infty$.

The proof follows an argument very much similar to that used in the proof of my former Theorem II. The underlying idea is due essentially to M. and F. Riesz; these authors dealt, of course, only with the inequality (1) of $\mathrm{S}$. Bernstein. In the present case the chief difficulty is to prove the positivity of the trigonometric polynomial

$$
\begin{aligned}
M_{n}(\theta)=1+2 \sum_{\nu=1}^{n-1}\left\{\frac{\rho_{n-\nu}}{\rho_{n}} \cos n \theta \cos (\right. & -\nu) \theta \\
& +[(n-\nu) / n] \sin n \theta \sin (n-\nu) \theta\}
\end{aligned}
$$

for all real values of $\theta$.

Unfortunately, my proof for (3) fails if $n=2$ or $n=3$. In these cases the less precise inequality

$$
|\operatorname{grad} u| \leqq 2^{1 / 2} n
$$

can be obtained. We notice that $2^{1 / 2} \cdot 2=2.82 \cdots$ whereas $\rho_{2}=8 / 3=2.66 \cdots$, and $2^{1 / 2} \cdot 3=4.23 \cdots$ whereas $\rho_{3}=21 / 5=4.20$.

\section{FIRST PART OF THE PROOF}

1. It is sufficient to prove (3) for $x=y=0, z=1$. For the other points of the unit sphere the statement follows by a simple rotation, for the interior points by considering $u(k x, k y, k z), 0 \leqq k<1$.

If we introduce polar coordinates $r, \theta, \phi$ (in the usual notation), we have for $u(x, y, z)=U(r, \theta, \phi)$ 
(5)

$$
|\operatorname{grad} u|=\left\{U_{r}^{2}+\left(r^{-1} U_{\theta}\right)^{2}+\left((r \sin \theta)^{-1} U_{\phi}\right)^{2}\right\}^{1 / 2},
$$

so that our inequality (3) is equivalent to the following statement: Let $\alpha, \beta$ be arbitrary real constants, and

$$
K=\cos \alpha \cdot U_{r}+\sin \alpha \cos \beta \cdot r^{-1} U_{\theta}+\sin \alpha \sin \beta(r \sin \theta)^{-1} U_{\phi} ;
$$

then

(7)

$$
|K| \leqq \rho_{n} .
$$

2. Representing $u(x, y, z)=U(r, \theta, \phi)$ in the form

$$
U(r, \theta, \phi)=A_{0}(r, \theta)+\sum_{\nu=1}^{n}\left(A_{\nu}(r, \theta) \cos \nu \phi+B_{\nu}(r, \theta) \sin \nu \phi\right),
$$

we have

$$
\begin{aligned}
& A_{\nu}(r, \theta)=\sin ^{\nu} \theta \sum_{\mu=\nu}^{n} a_{\mu \nu} P_{\mu}^{(\nu)}(\cos \theta) r^{\mu} \\
& B_{\nu}(r, \theta)=\sin ^{\nu} \theta \sum_{\mu=\nu}^{n} b_{\mu \nu} P_{\mu}^{(\nu)}(\cos \theta) r^{\mu}
\end{aligned}
$$

where $a_{\mu \nu}$ and $b_{\mu \nu}$ are real constants; $P_{\mu}(\cos \theta)$ is Legendre's polynomial in the usual notation.

For $x=y=0, z=1$, or $r=1, \theta=0$, we find

$$
\begin{aligned}
U_{r} & =\frac{\partial A_{0}}{\partial r}=\sum_{\mu=1}^{n} \mu a_{\mu 0}, \\
r^{-1} U_{\theta} & =\frac{\partial A_{1}}{\partial \theta} \cos \phi+\frac{\partial B_{1}}{\partial \theta} \sin \phi \\
& =\sum_{\mu=1}^{n} P_{\mu}^{\prime}(1)\left(a_{\mu 1} \cos \phi+b_{\mu 1} \sin \phi\right),
\end{aligned}
$$

$$
\begin{aligned}
\lim _{\theta \rightarrow 0}\left((r \sin \theta)^{-1} U_{\phi}\right) & =-\frac{\partial A_{1}}{\partial \theta} \sin \phi+\frac{\partial B_{1}}{\partial \theta} \cos \phi \\
& =\sum_{\mu=1}^{n} P_{\mu}^{\prime}(1)\left(-a_{\mu 1} \sin \phi+b_{\mu 1} \cos \phi\right),
\end{aligned}
$$

so that

$$
\begin{aligned}
K=\cos \alpha \sum_{\mu=1}^{n} \mu a_{\mu 0}+\sin \alpha \cdot \cos (\phi+\beta) \sum_{\mu=1}^{n} P_{\mu}^{\prime}(1) a_{\mu 1} \\
\\
\quad+\sin \alpha \sin (\phi+\beta) \sum_{\mu=1}^{n} P_{\mu}^{\prime}(1) b_{\mu 1}
\end{aligned}
$$


3. According to the orthogonality property of the surface harmonics, we have

$$
\begin{gathered}
\int_{0}^{\pi} \int_{-\pi}^{+\pi} U\left(1, \theta^{\prime}, \phi^{\prime}\right) P_{\mu}\left(\cos \theta^{\prime}\right) \sin \theta^{\prime} d \theta^{\prime} d \phi^{\prime}=\frac{4 \pi}{2 \mu+1} a_{\mu 0}, \\
\int_{0}^{\pi} \int_{-\pi}^{+\pi} U\left(1, \theta^{\prime}, \phi^{\prime}\right) \sin \theta^{\prime} P_{\mu}^{\prime}\left(\cos \theta^{\prime}\right) \begin{array}{l}
\cos \phi^{\prime} \\
\sin \phi^{\prime}
\end{array} \sin \theta^{\prime} d \theta^{\prime} d \phi^{\prime}=\frac{4 \pi}{2 \mu+1} \cdot\left\{\begin{array}{l}
P_{\mu}^{\prime}(1) a_{\mu 1}, \\
P_{\mu}^{\prime}(1) b_{\mu 1},
\end{array}\right.
\end{gathered}
$$

so that

$$
\begin{aligned}
K=\frac{1}{4 \pi} & \int_{0}^{\pi} \int_{-\pi}^{+\pi} U\left(1, \theta^{\prime}, \phi^{\prime}\right)\left\{\cos \alpha \sum_{\mu=1}^{n} \mu(2 \mu+1) P_{\mu}\left(\cos \theta^{\prime}\right)\right. \\
& \left.\quad+\sin \alpha \cos \left(\phi-\phi^{\prime}+\beta\right) \sum_{\mu=1}^{n}(2 \mu+1) \sin \theta^{\prime} P_{\mu}^{\prime}\left(\cos \theta^{\prime}\right)\right\} \sin \theta^{\prime} d \theta^{\prime} d \phi^{\prime} .
\end{aligned}
$$

In these integrals, $0 \leqq \theta^{\prime} \leqq \pi,-\pi \leqq \phi^{\prime} \leqq+\pi$.

4. Now, we introduce the Fourier expansions

$$
\begin{aligned}
\frac{1}{4} \pi \sin \theta \sum_{\mu=1}^{n} \mu(2 \mu+1) & P_{\mu}(\cos \theta) \\
& =\rho_{1} \cos \theta+\rho_{2} \cos 2 \theta+\cdots+\rho_{n} \cos n \theta+\cdots, \\
\frac{1}{8} \pi \sin ^{2} \theta \sum_{\mu=1}^{n}(2 \mu+1) & P_{\mu}^{\prime}(\cos \theta) \\
& =\sigma_{1} \sin \theta+\sigma_{2} \sin 2 \theta+\cdots+\sigma_{n} \sin n \theta+\cdots .
\end{aligned}
$$

Since

$$
\int_{0}^{\pi} \sin \theta P_{\mu}(\cos \theta) \cos \nu \theta d \theta=\int_{0}^{\pi} \sin ^{2} \theta P_{\mu}^{\prime}(\cos \theta) \sin \nu \theta d \theta=0, \quad \mu>\nu,
$$

the coefficients $\rho_{\nu}, \sigma_{\nu}, 1 \leqq \nu \leqq n$, depend only on $\nu$; that is to say, for a fixed value of $\nu$, they are independent of $n$. Consequently, we find from (11):

$$
\begin{aligned}
K=\frac{1}{2 \pi^{2}} \int_{-\pi}^{+\pi} \int_{-\pi}^{+\pi} U\left(1, \theta^{\prime}, \phi^{\prime}\right) & \left\{\cos \alpha \sum_{\nu=1}^{n} \rho_{\nu} \cos \nu \theta^{\prime}\right. \\
& \left.+2 \sin \alpha \cos \left(\phi-\phi^{\prime}+\beta\right) \sum_{\nu=1}^{n} \sigma_{\nu} \sin \nu \theta^{\prime}\right\} d \theta^{\prime} d \phi^{\prime} .
\end{aligned}
$$

Here both $\theta^{\prime}, \phi^{\prime}$ run from $-\pi$ to $+\pi$; we use the fact that

$$
\int_{-\pi}^{+\pi} U\left(1, \theta^{\prime}, \phi^{\prime}\right) d \phi^{\prime}
$$


is a cosine polynomial of the $n$th degree, and also that both integrals

$$
\int_{-\pi}^{+\pi} U\left(1, \theta^{\prime}, \phi^{\prime}\right) \cos \phi^{\prime} \sin \phi^{\prime} d \phi^{\prime}
$$

represent sine polynomials of the $n$th degree.

\section{SECOND PART OF THE PROOF}

The basic idea of this part is similar to that used in the proof of II (see the Introduction).

1. We write (13) in the form

$$
\begin{aligned}
K=\frac{1}{2 \pi^{2}} \int_{-\pi}^{+\pi} \int_{-\pi}^{+\pi} U\left(1, \theta^{\prime}, \phi^{\prime}\right)\{ & \cos \alpha \cdot \rho\left(\theta^{\prime}\right) \\
& \left.+2 \sin \alpha \cos \left(\phi-\phi^{\prime}+\beta\right) \cdot \sigma\left(\theta^{\prime}\right)\right\} d \theta^{\prime} d \phi^{\prime}
\end{aligned}
$$

where

$$
\rho\left(\theta^{\prime}\right)=\sum_{\nu=1}^{n} \rho_{\nu} \cos \nu \theta^{\prime}, \quad \sigma\left(\theta^{\prime}\right)=\sum_{\nu=1}^{n} \sigma_{\nu} \sin \nu \theta^{\prime}
$$

Let us put

(15) $\rho_{n} \cos \alpha \cos n \theta^{\prime}+2 \sigma_{n} \sin \alpha \cos \left(\phi-\phi^{\prime}+\beta\right) \sin n \theta^{\prime}=R \cos \left(n \theta^{\prime}-\delta\right)$, where $R>0, \delta$ real, and $R, \delta$ are independent of $\theta^{\prime}$; obviously, $R$ and $\delta$ depend on $\alpha, \beta, \phi, \phi^{\prime}, n$. We have

$$
R=\left\{\rho_{n}^{2} \cos ^{2} \alpha+4 \sigma_{n}^{2} \sin ^{2} \alpha \cos ^{2}\left(\phi-\phi^{\prime}+\beta\right)\right\}^{1 / 2} .
$$

Furthermore, for $1 \leqq \nu \leqq n-1$,

$$
\begin{gathered}
\rho_{n-\nu} \cos \alpha \cos (n-\nu) \theta^{\prime}+2 \sigma_{n-\nu} \sin \alpha \cos \left(\phi-\phi^{\prime}+\beta\right) \sin (n-\nu) \theta^{\prime} \\
=\left(\rho_{n-\nu} / \rho_{n}\right) R \cos \delta \cos \left\{\left(n \theta^{\prime}-\delta\right)-\left(\nu \theta^{\prime}-\delta\right)\right\} \\
\quad+\left(\sigma_{n-\nu} / \sigma_{n}\right) R \sin \delta \sin \left\{\left(n \theta^{\prime}-\delta\right)-\left(\nu \theta^{\prime}-\delta\right)\right\} .
\end{gathered}
$$

Adding to this expression

$$
\begin{aligned}
\left(\rho_{n-\nu} / \rho_{n}\right) R \cos \delta \cos \left\{\left(n \theta^{\prime}-\delta\right)\right. & \left.+\left(\nu \theta^{\prime}-\delta\right)\right\} \\
& -\left(\sigma_{n-\nu} / \sigma_{n}\right) R \sin \delta \sin \left\{\left(n \theta^{\prime}-\delta\right)+\left(\nu \theta^{\prime}-\delta\right)\right\},
\end{aligned}
$$

we obtain

$2 R \cos \left(n \theta^{\prime}-\delta\right)\left\{\left(\rho_{n-\nu} / \rho_{n}\right) \cos \delta \cos \left(\nu \theta^{\prime}-\delta\right)-\left(\sigma_{n-v} / \sigma_{n}\right) \sin \delta \sin \left(\nu \theta^{\prime}-\delta\right)\right\}$.

Consequently, since $U\left(1, \theta^{\prime}, \phi^{\prime}\right)$ is a trigonometric polynomial of the $n$th degree in $\theta^{\prime}$, 


$$
\begin{aligned}
K= & \frac{1}{2 \pi^{2}} \int_{-\pi}^{+\pi} \int_{-\pi}^{+\pi} U\left(1, \theta^{\prime}, \phi^{\prime}\right) \cdot R \cos \left(n \theta^{\prime}-\delta\right) \\
& \cdot\left\{1+2 \sum_{\nu=1}^{n-1} \frac{\rho_{n-\nu}}{\rho_{n}} \cos \delta \cos \left(\nu \theta^{\prime}-\delta\right)-\frac{\sigma_{n-\nu}}{\sigma_{n}} \sin \delta \sin \left(\nu \theta^{\prime}-\delta\right)\right\} d \theta^{\prime} d \phi^{\prime} .
\end{aligned}
$$

2. We now replace $\cos \left(n \theta^{\prime}-\delta\right)$ by

$$
\frac{1}{4 r}\left\{\frac{1-r^{2}}{1-2 r \cos \left(n \theta^{\prime}-\delta\right)+r^{2}}-\frac{1-r^{2}}{1+2 r \cos \left(n \theta^{\prime}-\delta\right)+r^{2}}\right\}, \quad 0<r<1,
$$

and obtain in the usual manner*

$$
\begin{aligned}
K= & \lim _{r \rightarrow 1-0} \frac{1}{8 \pi^{2} r} \int_{-\pi}^{+\pi} \int_{-\pi}^{+\pi} U\left(1, \theta^{\prime}, \phi^{\prime}\right) \\
& \cdot R\left\{\frac{1-r^{2}}{1-2 r \cos \left(n \theta^{\prime}-\delta\right)+r^{2}}-\frac{1-r^{2}}{1+2 r \cos \left(n \theta^{\prime}-\delta\right)+r^{2}}\right\} \\
& \cdot\left\{1+2 \sum_{\nu=1}^{n-1}\left(\frac{\rho_{n-\nu}}{\rho_{n}} \cos \delta \cos \left(\nu \theta^{\prime}-\delta\right)\right.\right. \\
= & \frac{1}{4 \pi n} \int_{-\pi}^{+\pi} \sum^{\prime} U\left(1, \theta^{\prime}, \phi^{\prime}\right) R\left\{1+2 \sum_{\nu=1}^{n-1}\left(\frac{\rho_{n-\nu}}{\rho_{n}} \cos \delta \cos \left(\nu \theta^{\prime}-\delta\right)\right.\right. \\
& \left.\left.-\frac{\sigma_{n-\nu}}{\sigma_{n}} \sin \delta \sin \left(\nu \theta^{\prime}-\delta\right)\right)\right\} d \theta^{\prime} d \phi^{\prime} \\
& \left.\left.-\frac{1}{\sigma_{n}} \sin \delta \sin \left(\nu \theta^{\prime}-\delta\right)\right)\right\} d \phi^{\prime} \\
\int_{-\pi}^{+\pi} \sum^{\prime \prime} U\left(1, \theta^{\prime}, \phi^{\prime}\right) R\left\{1+2 \sum_{\nu=1}^{n-1}\left(\frac{\rho_{n-\nu}}{\rho_{n}} \cos \delta \cos \left(\nu \theta^{\prime}-\delta\right)\right.\right. & \left.\left.-\frac{\sigma_{n-\nu}}{\sigma_{n}} \sin \delta \sin \left(\nu \theta^{\prime}-\delta\right)\right)\right\} d \phi^{\prime}
\end{aligned}
$$

The summations $\sum^{\prime}, \sum^{\prime \prime}$ are extended over the modulo $2 \pi$ incongruent values of $\theta^{\prime}$ for which $n \theta^{\prime}-\delta \equiv 0$ and $\pi$, respectively. (These particular values of $\theta^{\prime}$ depend, of course, on $\alpha, \beta, \phi, \phi^{\prime}, n$.) For these values

$$
\begin{aligned}
1 & +2 \sum_{\nu=1}^{n-1}\left(\frac{\rho_{n-\nu}}{\rho_{n}} \cos \delta \cos \left(\nu \theta^{\prime}-\delta\right)-\frac{\sigma_{n-\nu}}{\sigma_{n}} \sin \delta \sin \left(\nu \theta^{\prime}-\delta\right)\right) \\
& =1+2 \sum_{\nu=1}^{n-1}\left(\frac{\rho_{n-\nu}}{\rho_{n}} \cos n \theta^{\prime} \cos (n-\nu) \theta^{\prime}+\frac{\sigma_{n-\nu}}{\sigma_{n}} \sin n \theta^{\prime} \sin (n-\nu) \theta^{\prime}\right)=M_{n}\left(\theta^{\prime}\right)
\end{aligned}
$$

* Szegö, loc. cit., p. 64. 
Since $\sigma_{n}=n$ (see (29)), this is the same trigonometric polynomial of order $2 n-1$ as defined in (4). It depends only on $n$.

Consequently,

$$
\begin{aligned}
K=\frac{1}{4 \pi n} \int_{-\pi}^{+\pi} \sum^{\prime} U\left(1, \theta^{\prime}, \phi^{\prime}\right) R M_{n}\left(\theta^{\prime}\right) d \phi^{\prime} \\
\\
\quad-\frac{1}{4 \pi n} \int_{-\pi}^{+\pi} \sum^{\prime \prime} U\left(1, \theta^{\prime}, \phi^{\prime}\right) R M_{n}\left(\theta^{\prime}\right) d \phi^{\prime} .
\end{aligned}
$$

Here and in the next section, $\sum^{\prime}$ and $\sum^{\prime \prime}$ have the meaning given above.

3. In the last part of the proof we are going to show that $M_{n}\left(\theta^{\prime}\right)>0$ for all real values of $\theta^{\prime}$. Anticipating this result, we obtain from (19)

$$
|K| \leqq \frac{1}{4 \pi n} \int_{-\pi}^{+\pi} \sum^{\prime} R M_{n}\left(\theta^{\prime}\right) d \phi^{\prime}+\frac{1}{4 \pi n} \int_{-\pi}^{+\pi} \sum^{\prime \prime} R M_{n}\left(\theta^{\prime}\right) d \phi^{\prime} .
$$

But we easily see that the trigonometric polynomial $M_{n}\left(\theta^{\prime}\right)$ does not contain any term with $\cos n \theta^{\prime}$ and $\sin n \theta^{\prime}$, and also that its absolute term is 1 . Hence

$$
\frac{1}{n} \sum^{\prime} M_{n}\left(\theta^{\prime}\right)=\frac{1}{n} \sum^{\prime \prime} M_{n}\left(\theta^{\prime}\right)=1,
$$

so that on account of (16),

$$
\begin{aligned}
|K| & \leqq \frac{1}{4 \pi} \int_{-\pi}^{+\pi} R d \phi^{\prime}+\frac{1}{4 \pi} \int_{-\pi}^{+\pi} R d \phi^{\prime} \\
& =\frac{1}{2 \pi} \int_{-\pi}^{+\pi}\left(\rho_{n}^{2} \cos ^{2} \alpha+4 \sigma_{n}^{2} \sin ^{2} \alpha \cos ^{2} \phi^{\prime}\right)^{1 / 2} d \phi^{\prime} .
\end{aligned}
$$

By means of Schwarz's inequality

$$
K^{2} \leqq \frac{1}{2 \pi} \int_{-\pi}^{+\pi}\left(\rho_{n}^{2} \cos ^{2} \alpha+4 \sigma_{n}^{2} \sin ^{2} \alpha \cos ^{2} \phi^{\prime}\right) d \phi^{\prime}=\rho_{n}{ }^{2} \cos ^{2} \alpha+2 \sigma_{n}^{2} \sin ^{2} \alpha \leqq \rho_{n}{ }^{2},
$$

provided that $\rho_{n}^{2} \geqq 2 \sigma_{n}^{2}$. This is the case for $n \geqq 4$ (see (30)). For these values of $n$, the inequality $|K| \leqq \rho_{n}$ is proved.

4. The preceding argument, combined with (30), shows that $|K|<\rho_{n}$ holds provided that $\sin ^{2} \alpha>0$. Now let $\sin ^{2} \alpha=0, \cos \alpha=1$; then $\delta=0, R=\rho_{n}$. In this case (19) reads as follows:

$$
K=\frac{\rho_{n}}{2 n} \sum^{\prime} U\left(\theta^{\prime}\right) M_{n}\left(\theta^{\prime}\right)-\frac{\rho_{n}}{2 n} \sum^{\prime \prime} U\left(\theta^{\prime}\right) M_{n}\left(\theta^{\prime}\right) .
$$

Here $U\left(\theta^{\prime}\right)=(2 \pi)^{-1} \int_{-\pi}^{+\pi} U\left(1, \theta^{\prime}, \phi^{\prime}\right) d \phi^{\prime}$, and the sums $\sum^{\prime}, \sum^{\prime \prime}$ are extended 
over the modulo $2 \pi$ incongruent solutions of the equations $n \theta^{\prime} \equiv 0$ and $\pi$, respectively. Taking (21) into consideration, we see that $K=\rho_{n}$ holds if and only if $U\left(\theta^{\prime}\right)=\cos n \theta^{\prime}$ for the special values of $\theta^{\prime}$ mentioned. Since the cosine polynomial $U\left(\theta^{\prime}\right)-\cos n \theta^{\prime}$ vanishes for the values $\theta^{\prime}=0, \pi / n, 2 \pi / n, \cdots, \pi$ (for which $\cos \theta^{\prime}$ assumes $n+1$ distinct values), it must vanish identically, that is, $U(\theta) \equiv \cos n \theta$.

Similarly $K=-\rho_{n}$ holds if and only if $U(\theta)=-\cos n \theta$.

Considering the case $U(\theta)=\cos n \theta$, we write again $\theta=\theta^{\prime}=k \pi / n, k$ an integer. This furnishes

$$
\frac{1}{2 \pi} \int_{-\pi}^{+\pi} U\left(1, \theta^{\prime}, \phi^{\prime}\right) d \phi^{\prime}=(-1)^{k}
$$

Since $\left|U\left(1, \theta^{\prime}, \phi^{\prime}\right)\right| \leqq 1$, we find $U\left(1, \theta^{\prime}, \phi^{\prime}\right)=(-1)^{k}$ identically in $\phi^{\prime}$. Consequently, by use of the notation (8), (9), we have for the values of $\theta^{\prime}$ mentioned before, $A_{\nu}\left(1, \theta^{\prime}\right)=B_{\nu}\left(1, \theta^{\prime}\right)=0, \nu=1,2, \cdots, n$, or if $\sin \theta^{\prime} \neq 0$,

$$
\sum_{\mu=\nu}^{n} a_{\mu \nu} P_{\mu}^{(\nu)}\left(\cos \theta^{\prime}\right)=\sum_{\mu=\nu}^{n} b_{\mu \nu} P_{\mu}^{(\nu)}\left(\cos \theta^{\prime}\right)=0 .
$$

These equations hold in particular for $\theta^{\prime}=\pi / n, 2 \pi / n, \cdots,(n-1) \pi / n$, so that $A_{\nu}(1, \theta)$ and $B_{\nu}(1, \theta)$ must vanish identically for $\nu$ greater than or equal to 2 , and $A_{1}(1, \theta)=$ const. $\sin n \theta, B_{\nu}(1, \theta)=$ const. $\sin n \theta$. Finally, $U(1, \theta, \phi)=\cos n \theta$ $+c \sin n \theta \cos \left(\phi-\phi_{0}\right), c$ and $\phi_{0}$ constant, and as a consequence of $|U| \leqq 1$ we find $c=0$.

\section{Calculation of $\rho_{n}$ AND $\sigma_{n}$}

1. The constants $\rho_{n}$ and $\sigma_{n}$ involved in the previous considerations, can be calculated in various ways. A comparatively simple procedure is based on the well known formulas

$$
\begin{aligned}
& \lim _{\epsilon \rightarrow+0}\left\{Q_{n}(u+i \epsilon)-Q_{n}(u-i \epsilon)\right\}=-i \pi P_{n}(u), \\
& \lim _{\epsilon \rightarrow+0}\left\{Q_{n}^{\prime}(u+i \epsilon)-Q_{n}^{\prime}(u-i \epsilon)\right\}=-i \pi P_{n}^{\prime}(u),
\end{aligned}
$$

where $-1<u<+1$ and $Q_{n}(\xi)$ denotes Legendre's function of the second kind. Let $z=r e^{i \theta}, 0<r<1,0<\theta<\pi$; then (23) can be written as follows:

$$
\begin{gathered}
P_{n}(\cos \theta)=\frac{2}{\pi} \lim _{r \rightarrow 1-0} \Im Q_{n}\left(\frac{1}{2}\left(z+z^{-1}\right)\right), \\
P_{n}^{\prime}(\cos \theta)=\frac{2}{\pi} \lim _{r \rightarrow 1-0} \Im Q_{n}^{\prime}\left(\frac{1}{2}\left(z+z^{-1}\right)\right),
\end{gathered}
$$

so that 
(25)

$$
\begin{aligned}
\frac{1}{4} \pi \sin \theta \sum_{\mu=1}^{n} \mu(2 \mu+1) P_{\mu}(\cos \theta) & \\
= & \lim _{r \rightarrow 1-0} \frac{\pi}{4} \frac{z-z^{-1}}{2 i} \frac{2}{\pi} \Im \sum_{\mu=1}^{n} \mu(2 \mu+1) Q_{\mu}\left(\frac{1}{2}\left(z+z^{-1}\right)\right) \\
& =\lim _{r \rightarrow 1-0} \Re\left\{\frac{1-z^{2}}{4 z} \sum_{\mu=1}^{n} \mu(2 \mu+1) Q_{\mu}\left(\frac{1}{2}\left(z+z^{-1}\right)\right)\right\},
\end{aligned}
$$

$$
\begin{aligned}
\frac{1}{8} \pi \sin ^{2} \theta \sum_{\mu=0}^{n} & (2 \mu+1) P_{\mu}^{\prime}(\cos \theta) \\
& =\lim _{r \rightarrow 1-0} \frac{\pi}{8}\left(\frac{z-z^{-1}}{2 i}\right)^{2} \frac{2}{\pi} \Im \sum_{\mu=0}^{n}(2 \mu+1) Q_{\mu}^{\prime}\left(\frac{1}{2}\left(z+z^{-1}\right)\right) \\
& =-\lim _{r \rightarrow 1-0} \Im\left\{\frac{\left(1-z^{2}\right)^{2}}{16 z^{2}} \sum_{\mu=0}^{n}(2 \mu+1) Q_{\mu}^{\prime}\left(\frac{1}{2}\left(z+z^{-1}\right)\right)\right\} .
\end{aligned}
$$

Now $Q_{\mu}\left(\left(z+z^{-1}\right) / 2\right)$ is a power series of $z$ starting with $z^{\mu+1}$; therefore $z^{-2} Q_{\mu}{ }^{\prime}\left(\left(z+z^{-1}\right) / 2\right)$ is a power series starting with $z^{\mu}$. Adding to the sums on the right-hand side of (25) the corresponding terms with $\mu>n$, we do not influence the coefficients of $z, z^{2}, \cdots, z^{n}$. From this, we conclude that the $n$ first coefficients of the expansions (12) agree with the corresponding coefficients of

$$
\begin{aligned}
& f(z)=\frac{1-z^{2}}{4 z} \sum_{\mu=1}^{\infty} \mu(2 \mu+1) Q_{\mu}\left(\frac{1}{2}\left(z+z^{-1}\right)\right), \\
& g(z)=-\frac{\left(1-z^{2}\right)^{2}}{16 z^{2}} \sum_{\mu=0}^{\infty}(2 \mu+1) Q_{\mu}^{\prime}\left(\frac{1}{2}\left(z+z^{-1}\right)\right) .
\end{aligned}
$$

In particular, $\rho_{n}$ and $\sigma_{n}$ are the coefficients of $z^{n}$ in the power series expansions of the functions (26), regular for $|z|<1$.

2. In order to compute the functions (26), we start from the classical formula

$$
\begin{aligned}
\frac{1}{\xi-1} & =\sum_{\mu=0}^{n}(2 \mu+1) Q_{\mu}(\xi)+\sum_{\mu=n+1}^{\infty}(2 \mu+1) Q_{\mu}(\xi) \\
& =\sum_{\mu=0}^{n}(2 \mu+1) Q_{\mu}(\xi)+\frac{n+1}{\xi-1}\left\{Q_{n}(\xi)-Q_{n+1}(\xi)\right\} \\
& =\sum_{\mu=0}^{n}(2 \mu+1) Q_{\mu}(\xi)+r_{n+1}(\xi) .
\end{aligned}
$$

Here $\xi=\left(z+z^{-1}\right) / 2,|z|<1$, is in the complex plane cut along the segment $[-1,+1]$. Now 


$$
\begin{aligned}
\sum_{\mu=1}^{\infty} \mu(2 \mu+1) Q_{\mu}(\xi) & =\sum_{\mu=1}^{\infty} \mu\left(r_{\mu}(\xi)-r_{\mu+1}(\xi)\right)=r_{1}(\xi)+r_{2}(\xi)+r_{3}(\xi)+\cdots \\
& =\frac{1}{\xi-1} \sum_{\mu=1}^{\infty} \mu\left(Q_{\mu-1}(\xi)-Q_{\mu}(\xi)\right)=\frac{1}{\xi-1} \sum_{\mu=0}^{\infty} Q_{\mu}(\xi)
\end{aligned}
$$

so that from the well known integral representation of $Q_{\mu}(\xi)$, by use of the generating function of $P_{\mu}(t)$,

$$
\begin{aligned}
\sum_{\mu=1}^{\infty} \mu(2 \mu+1) Q_{\mu}(\xi) & =\frac{1}{\xi-1} \sum_{\mu=0}^{\infty} \frac{1}{2} \int_{-1}^{+1} \frac{P_{\mu}(t)}{\xi-t} d t=\frac{2^{-3 / 2}}{\xi-1} \int_{-1}^{+1} \frac{(1-t)^{-1 / 2}}{\xi-t} d t \\
& =\frac{2^{-1 / 2}}{(\xi-1)^{3 / 2}} \arctan \left(\frac{2}{\xi-1}\right)^{1 / 2} \\
& =\frac{2 z^{3 / 2}}{(1-z)^{3}} \arctan \left(\frac{2 z^{1 / 2}}{1-z}\right) \\
& =\frac{4 z^{3 / 2}}{(1-z)^{3}} \arctan \left(z^{1 / 2}\right) .
\end{aligned}
$$

Consequently,

$$
\begin{aligned}
f(z)=\frac{1+z}{(1-z)^{2}} z^{1 / 2} \arctan \left(z^{1 / 2}\right) & =\frac{1+z}{(1-z)^{2}} \frac{z^{1 / 2}}{2 i} \log \frac{1+i z^{1 / 2}}{1-i z^{1 / 2}} \\
& =\sum_{\nu=0}^{\infty}(2 \nu+1) z^{\nu} \cdot \sum_{\nu=1}^{\infty} \frac{(-1)^{\nu-1}}{2 \nu-1} z^{\nu},
\end{aligned}
$$

$$
\begin{aligned}
\rho_{n} & =\sum_{\nu=1}^{n} \frac{(-1)^{\nu-1}}{2 \nu-1}(2 n-2 \nu+1) \\
& =2 n\left(\frac{1}{1}-\frac{1}{3}+\cdots+\frac{(-1)^{n-1}}{2 n-1}\right)+\left\{\begin{aligned}
0, & n \text { even }, \\
-1, & n \text { odd },
\end{aligned}\right.
\end{aligned}
$$

which agrees with the expression in the right-hand member of the inequality (3).

3. A simpler argument furnishes from (27)

$$
\begin{gathered}
\sum_{\mu=0}^{\infty}(2 \mu+1) Q_{\mu}^{\prime}(\xi)=-(\xi-1)^{-2}=-4 z^{2}(1-z)^{-4}, \\
g(z)=\frac{1}{4}\left(\frac{1+z}{1-z}\right)^{2}, \sigma_{n}=n .
\end{gathered}
$$

4. It can be easily seen that

$$
\rho_{n}>2^{1 / 2} \sigma_{n}
$$
for $n \geqq 4$. 
Indeed, we write

$$
\frac{\rho_{n}}{2 n}=1-\frac{1}{3}+\cdots+\frac{(-1)^{n-1}}{2 n-1}+\frac{\epsilon_{n}}{2 n},
$$

where $\epsilon_{n}=0$ or -1 , according as $n$ is even or odd. If $n$ is even, we find, $n \geqq 4$,

$$
\frac{\rho_{n}}{2 n} \geqq 1-\frac{1}{3}+\frac{1}{5}-\frac{1}{7}=\frac{76}{105}>2^{-1 / 2} .
$$

If $n$ is odd, $n \geqq 5$,

$$
\frac{\rho_{n}}{2 n}=\frac{\rho_{n-1}}{2(n-1)}+\frac{1}{2 n-1}-\frac{1}{2 n}>\frac{\rho_{n-1}}{2(n-1)}>2^{-1 / 2} .
$$

Apparently, (30) does not hold for $n=1,2,3$. Indeed $\rho_{1}=1, \rho_{2}=8 / 3$, $\rho_{3}=21 / 5, \sigma_{1}=1, \sigma_{2}=2, \sigma_{3}=3$.

\section{Positivity of $M_{n}(\theta)$}

Let $n \geqq 2$. The remaining part of the proof is devoted to the discussion of the trigonometric polynomial (4), that is, of

$$
M_{n}(\theta)=2 \sum_{\nu=0}^{n-1} *\left(\frac{\rho_{n-\nu}}{\rho_{n}} \cos n \theta \cos (n-\nu) \theta+\frac{n-\nu}{n} \sin n \theta \sin (n-\nu) \theta\right),
$$

where the symbol $\sum^{*}$ indicates that the term $\nu=0$ must be multiplied by $1 / 2$. We want to show that $M_{n}(\theta)>0$.

1. Let $0<\theta<\pi$. For the function $f(z)$ in (28), we have $f(0)=0$, so that

$$
\begin{aligned}
2 \sum_{\nu=0}^{n-1} \rho_{n-\nu} \cos (n-\nu) \theta & =\frac{1}{2 \pi i} \int \frac{f(z)}{z^{n+1}}\left\{2 \sum_{\nu=0}^{n-1} z^{\nu} \cos (n-\nu) \theta\right\} d z \\
& =\frac{1}{2 \pi i} \int \frac{f(z)}{z^{n+1}}\left\{\cos n \theta+2 \sum_{\nu=1}^{\infty} z^{\nu} \cos (n-\nu) \theta\right\} d z \\
& =\frac{1}{2 \pi i} \int \frac{f(z)}{z^{n+1}} \frac{\left(1-z^{2}\right) \cos n \theta+2 z \sin n \theta \sin \theta}{1-2 z \cos \theta+z^{2}} d z
\end{aligned}
$$

In an arbitrary domain not containing $z=-1$, the function $f(z)$ has the only singularity $z=+1$; an additional singularity of the last integrand is at $z=e^{ \pm i \theta}$. The integrand being single-valued around $z=+1, e^{ \pm i \theta}$, we can choose as a contour the following: A "large" arc $|z|=R,|\arg z| \leqq \pi-\epsilon$, and (approximately) the segment $-R,-1$ of the negative real axis, counting twice and encircling $z=-1$ in the negative sense. The residues at $z=+1, e^{ \pm i \theta}$ have to be taken into consideration. 
Now, since $f(z)=O\left(|z|^{-1 / 2}\right.$ ) as $z \rightarrow \infty$ (compare the second form of $f(z)$ given in (28)), the contribution of the large arc can be disregarded. The residues furnish

$$
\begin{aligned}
& -\frac{d}{d z}\left\{(1+z) z^{-n-1 / 2} \arctan \left(z^{1 / 2}\right) \frac{\left(1-z^{2}\right) \cos n \theta+2 z \sin n \theta \sin \theta}{1-2 z \cos \theta+z^{2}}\right\}_{z=+1} \\
& -2 \Re\left\{e^{-i(n+1) \theta} f\left(e^{i \theta}\right) \frac{\left(1-e^{2 i \theta}\right) \cos n \theta+2 e^{i \theta} \sin n \theta \sin \theta}{e^{i \theta}-e^{-i \theta}}\right\} .
\end{aligned}
$$

The first term is

$$
\begin{gathered}
(-\pi / 4+(2 n+1) \pi / 4-1 / 2) \frac{2 \sin n \theta \sin \theta}{2(1-\cos \theta)}-\frac{\pi}{2} \frac{-2 \cos n \theta}{2(1-\cos \theta)} \\
=\frac{(n \pi-1) \sin n \theta \sin \theta+\pi \cos n \theta}{4 \sin ^{2}(\theta / 2)} .
\end{gathered}
$$

For the calculation of the second term, we note that for $0<\theta<\pi$

$$
\begin{aligned}
f\left(e^{i \theta}\right) & =\frac{1+e^{i \theta}}{\left(1-e^{i \theta}\right)^{2}} \frac{e^{i \theta / 2}}{2 i}\{\log \cot [(\theta+\pi) / 4]+i \pi / 2\} \\
& =\frac{\cos (\theta / 2)}{4 \sin ^{2}(\theta / 2)}\{i \log \cot [(\theta+\pi) / 4]-\pi / 2\},
\end{aligned}
$$

so that the second term of (32) is

$$
-2 \Re\left\{e^{-i(n+1) \theta} f\left(e^{i \theta}\right)\left(-e^{i(n+1) \theta}\right)\right\}=2 \Re f\left(e^{i \theta}\right)=\frac{-\pi \cos (\theta / 2)}{4 \sin ^{2}(\theta / 2)} .
$$

Finally, we consider

$$
\frac{1}{2 \pi i} \int_{-\infty}^{(-1,-)} \frac{f(z)}{z^{n+1}} \frac{\left(1-z^{2}\right) \cos n \theta+2 z \sin n \theta \sin \theta}{1-2 z \cos \theta+z^{2}} d z .
$$

On the "upper" border of the line $-\infty,-1$ we have, $z=-t, 1<t<+\infty$,

$$
f(z)=\frac{1-t}{(1+t)^{2}} \frac{t^{1 / 2}}{2}\left\{\log \left(\frac{t^{1 / 2}-1}{t^{1 / 2}+1}\right)+i \pi\right\},
$$

whereas on the "lower" border, $i \pi$ must be replaced by $-i \pi$. Consequently, (34) will be

$$
\frac{(-1)^{n}}{2} \int_{1}^{\infty} \frac{t-1}{(t+1)^{2}} t^{-n-1 / 2} \frac{\left(1-t^{2}\right) \cos n \theta-2 t \sin n \theta \sin \theta}{1+2 t \cos \theta+t^{2}} d t
$$

so that 


$$
\begin{aligned}
2 \sum_{\nu=0}^{n-1} * \rho_{n-\nu} & \cos (n-\nu) \theta=\frac{(n \pi-1) \sin n \theta \sin \theta+\pi \cos n \theta-\pi \cos (\theta / 2)}{4 \sin ^{2}(\theta / 2)} \\
+ & \frac{(-1)^{n}}{2} \int_{1}^{\infty} \frac{t-1}{(t+1)^{2}} t^{-n-1 / 2} \frac{\left(1-t^{2}\right) \cos n \theta-2 t \sin n \theta \sin \theta}{1+2 t \cos \theta+t^{2}} d t .
\end{aligned}
$$

2. According to a well known formula,

$$
\begin{aligned}
1+2 \sum_{\nu=1}^{n-1} \cos \nu \theta+\cos n \theta & =\frac{\sin (2 n-1)(\theta / 2)}{2 \sin (\theta / 2)}+\frac{\sin (2 n+1)(\theta / 2)}{2 \sin (\theta / 2)} \\
& =\sin n \theta \cot (\theta / 2),
\end{aligned}
$$

so that

$$
\begin{aligned}
2 \sum_{\nu=0}^{n-1} *(n-\nu) \sin (n-\nu) \theta & =2 \sum_{\nu=1}^{n-1} \nu \sin \nu \theta+n \sin n \theta \\
& =-n \cos n \theta \cot (\theta / 2)+\frac{\sin n \theta}{2 \sin ^{2}(\theta / 2)} .
\end{aligned}
$$

This leads, in view of (35), to the following important representation:

$$
\begin{aligned}
\rho_{n} M_{n}(\theta)= & \frac{(n \pi-1) \sin n \theta \sin \theta+\pi \cos n \theta-\pi \cos (\theta / 2)}{4 \sin ^{2}(\theta / 2)} \cos n \theta \\
& +\frac{(-1)^{n-1}}{2} \cos n \theta \int_{1}^{\infty} \frac{t-1}{(t+1)^{2}} t^{-n-1 / 2} \Re\left\{e^{i n \theta} \frac{1-t^{-1} e^{i \theta}}{1+t^{-1} e^{i \theta}}\right\} d t \\
& -\frac{1}{2} \rho_{n} \sin 2 n \theta \cot (\theta / 2)+\frac{\rho_{n}}{2 n} \frac{\sin ^{2} n \theta}{\sin ^{2}(\theta / 2)} .
\end{aligned}
$$

3. Now, we first establish the inequality

$$
\left|\frac{(-1)^{n-1}}{2} \cos n \theta \int_{1}^{\infty} \frac{t-1}{(t+1)^{2}} t^{-n-1 / 2} \Re\left\{e^{i n \theta} \frac{1-t^{-1} e^{i \theta}}{1+t^{-1} e^{i \theta}}\right\} d t\right|<\frac{1}{4 n} .
$$

Indeed,

$$
\left|\Re\left\{e^{i n \theta} \frac{1-t^{-1} e^{i \theta}}{1+t^{-1} e^{i \theta}}\right\}\right| \leqq \frac{1+t^{-1}}{1-t^{-1}},
$$

and

$$
\frac{1}{2} \int_{1}^{\infty} \frac{t^{-n-1 / 2}}{t+1} d t<\frac{1}{4} \int_{1}^{\infty} t^{-n-1} d t=\frac{1}{4 n}
$$

4. Next, we proceed to a discussion of the quantities $\rho_{n}$. If $s_{n}$ denotes the 
$n$th partial sum of the series $1 / 1-1 / 3+1 / 5-\cdots$, and $\epsilon_{n}=0$ or -1 according as $n$ is even or odd, we have

$$
\begin{aligned}
\rho_{n}= & 2 n s_{n}+\epsilon_{n}=2 n\left(\pi / 4-\sum_{\nu=n+1}^{\infty} \frac{(-1)^{\nu-1}}{2 \nu-1}\right)+\epsilon_{n} \\
= & n \pi / 2+\epsilon_{n}+(-1)^{n+1} 2 n\left\{\left(\frac{1}{2 n+1}-\frac{1}{2 n+3}\right)\right. \\
& \left.+\left(\frac{1}{2 n+5}-\frac{1}{2 n+7}\right)+\cdots\right\} .
\end{aligned}
$$

We compare the last series with .

$$
\frac{1}{2}\left(\frac{1}{2 n}-\frac{1}{2 n+4}\right)+\frac{1}{2}\left(\frac{1}{2 n+4}-\frac{1}{2 n+8}\right)+\cdots=\frac{1}{4 n} .
$$

Since

$$
\begin{aligned}
0 & <\frac{1}{2}\left(\frac{1}{2 n}-\frac{1}{2 n+4}\right)-\left(\frac{1}{2 n+1}-\frac{1}{2 n+3}\right) \\
& =\frac{6}{2 n(2 n+1)(2 n+3)(2 n+4)}<\frac{3}{8 n^{4}}
\end{aligned}
$$

we find

$$
\rho_{n}=n \pi / 2+\epsilon_{n}+(-1)^{n+1} / 2+\delta_{n}=\frac{1}{2}(n \pi-1)+\delta_{n},
$$

where

$$
\begin{aligned}
\left|\delta_{n}\right| & <\frac{3 n}{4}\left(\frac{1}{n^{4}}+\frac{1}{(n+2)^{4}}+\frac{1}{(n+4)^{4}}+\cdots\right) \\
& <\frac{3}{4} \frac{1}{n^{3}}+\frac{3}{8} n \int_{n}^{\infty} \frac{d x}{x^{4}}=\frac{3}{4} \frac{1}{n^{3}}+\frac{1}{8} \frac{1}{n^{2}} .
\end{aligned}
$$

Incidentally, $\operatorname{sgn} \delta_{n}=(-1)^{n}$.

5. Returning again to (36), we obtain

$$
\begin{aligned}
\rho_{n} M_{n}(\theta)= & \frac{(n \pi-1) \sin n \theta \sin \theta+\pi \cos n \theta-\pi \cos (\theta / 2)}{4 \sin ^{2}(\theta / 2)} \cos n \theta \\
& -\frac{1}{4}(n \pi-1) \sin 2 n \theta \cot (\theta / 2)+\frac{n \pi-1}{4 n} \frac{\sin ^{2} n \theta}{\sin ^{2}(\theta / 2)}+\delta_{n}^{\prime} \\
= & \frac{\pi(1-\cos (\theta / 2) \cos n \theta)-n^{-1} \sin ^{2} n \theta}{4 \sin ^{2}(\theta / 2)}+\delta_{n}^{\prime},
\end{aligned}
$$


where

$$
\begin{aligned}
\left|\delta_{n}^{\prime}\right| & <\frac{1}{4 n}+\left|\delta_{n}\right|\left(\frac{1}{2}|\sin 2 n \theta| \cot (\theta / 2)+\frac{1}{2 n} \frac{\sin ^{2} n \theta}{\sin ^{2}(\theta / 2)}\right) \\
& <\frac{1}{4 n}+\left|\delta_{n}\right|\left(\frac{1}{2} \cdot 2 n \theta \cdot \frac{2}{\theta}+\frac{1}{2 n} \cdot 4 n^{2}\right)=\frac{1}{4 n}+4 n\left|\delta_{n}\right|
\end{aligned}
$$

or, according to (39),

$$
\left|\delta_{n}^{\prime}\right|<3 /(4 n)+3 / n^{2} .
$$

6. In order to prove the positivity of (40), we distinguish two cases.

(a) Let $(1 / 2) n \pi \cos (\theta / 2)<1$. Then

$$
\pi(1-\lambda \cos (\theta / 2))-n^{-1}\left(1-\lambda^{2}\right),
$$

where $\lambda$ varies between -1 and +1 , has a minimum at $\lambda=(1 / 2) n \pi \cos (\theta / 2)$ which is $\pi-n^{-1}-(1 / 4) n \pi^{2} \cos ^{2}(\theta / 2)$. Therefore, we only have to show that

$$
\frac{\pi-n^{-1}-(1 / 4) n \pi^{2} \cos ^{2}(\theta / 2)}{4 \sin ^{2}(\theta / 2)}>\frac{3}{4 n}+\frac{3}{n^{2}} \text {. }
$$

Since $\pi-n^{-1}-n \pi^{2} / 4<0$, the expression on the left-hand side is a decreasing function of $\cos (\theta / 2)$, so that its minimum is attained for $\cos (\theta / 2)=2 / n \pi$. But

$$
\frac{\pi-n^{-1}-n^{-1}}{4\left(1-4 / n^{2} \pi^{2}\right)}=\frac{1}{4} \pi\left(1+\frac{2}{n \pi}\right)^{-1}>\frac{3}{4 n}+\frac{3}{n^{2}} ;
$$

this inequality is true for $n \geqq 3$.

(b) Let $(1 / 2) n \pi \cos (\theta / 2)>1$. Then (43) is decreasing and its minimum is reached for $\lambda=+1$, which is $\pi(1-\cos (\theta / 2))$. Therefore, it is sufficient to show that

$$
\frac{\pi(1-\cos (\theta / 2))}{4 \sin ^{2}(\theta / 2)}=\frac{\pi}{4(1+\cos (\theta / 2))} \geqq \frac{\pi}{8}>\frac{3}{4 n}+\frac{3}{n^{2}},
$$

which is true for $n \geqq 4$.

It remains to show that for $n=2$ and $n=3$, we have $\pi / 8>\left|\delta_{n}{ }^{\prime}\right|$, or using (41), $\pi / 8>1 /(4 n)+4 n\left|\delta_{n}\right|$. Since $\rho_{2}=8 / 3, \rho_{3}=21 / 5$, we find from (38) $0<\delta_{2}=19 / 6-\pi, 0<-\delta_{3}=3 \pi / 2-47 / 10$, so that the statements are $\pi>1$ $+64(19 / 6-\pi), \pi>2 / 3+96(3 \pi / 2-47 / 10)$. Both inequalities can easily be verified.

STANFORD UNIVERSITY,

Stanford University, Calif. 